\title{
A New Image Enhancement Algorithm via Wavelet Homomorphic Filtering Transform
}

\author{
Chao Li, Guangyao Li, Yunlan Tan, Xianglong Xu and \\ Huixian Duan
}

\begin{abstract}
A new spatial-frequency image enhancement algorithm via wavelet homomorphic filtering transform is proposed to enhance the contrast of an image. The wavelet analysis coefficients are processed using a high-pass filter to amplify the high spatial frequencies and attenuate the low spatial frequencies. So the object features can be emphasized while the undesired contributions within the image due to light source nonuniformity can be reduced. Experimental results show that this new algorithm can gain better performance in enhancing the local contrast of an image while maintaining its global appearance, in contrast to some representative algorithms.
\end{abstract}

Keywords. Image Enhancement, Homomorphic Filtering, Wavelet Transform.

2010 Mathematics Subject Classification. 68U10.

\section{Introduction}

The image enhancement is one of the basic contents and important techniques in image processing. Its goal is to improve the image quality so that the processed image is better than the original image for a specific application or set of objectives, and more suitable than the primitive image in human's visual characteristic or the machine recognition system [26]. It highlights certain special information of an image, simultaneously weakens or removes certain unnecessary information.

Although most image enhancement methods can improve the perception of information for human viewers to some extent, they still have some deficiencies, such as the loss of local contrast or the loss of details. Considering the existent problems in image processing, we propose a new image enhancement algorithm.

The paper is organized as follows: In Section 2, some related works are introduced. Then a new algorithm that applies homomorphic filtering based on the multi-scale representation is described in Section 3. We give an improved method of homomorphic filtering and the dyadic wavelet transform description. Further-

This work is supported by the National Key Technologies R\&D Program of China (863 Key Program); no. 2010AA122200. 
more, in Section 4, we grant experimental results. In Section 5 we compare our algorithm with some existing representative image enhancement methods. Finally, conclusions are drawn in Section 6.

\section{Related Work}

So far, plenty of techniques have been proposed. According to the survey of these available techniques $[6,7,31]$, the commonly used techniques of image enhancement can be divided into two broad categories: spatial domain methods and frequency domain methods. The spatial domain methods operate on image pixels directly. Many of these methods are based on gray-level histogram modifications $[8,28]$, while others are based on local contrast measures and edge information $[4,16]$, partial differential equations (PDE) $[13,32,36]$, etc. The particular Retinex approach has been introduced by Land and successfully applied to image dynamic range compression and image enhancement [12,17,19-21]. Retinex theory addressed the problem of separating the illumination from the reflectance in a given image and thereby compensation for non-uniform lighting. In order to solve the halo artifacts problem that usually exists in Retinex methods, low curvature image simplifier (LCIS) and the gradient domain method were proposed by Tumblin and Turk [34], and Fattal et al. [10], respectively. Marsi et al. [25] introduced an image enhancement method by using a recursive rational filter, which separated the image into illumination and reflectance, as in the Retinex. Majumder and Irani [24] presented the perception-based contrast enhancement method that maximizes the average local contrast of an image strictly constrained by a perceptual constraint derived directly from the Weber law. Panetta et al. [27] introduced a multi-histogram equalization method which utilizes the human visual system (HVS), and then extended the HVS-based multi-histogram equalization approach to create a general enhancement method. The frequency domain methods directly operate on the frequency domain. Polesel et al. [29] presented an adaptive unsharp masking for contrast enhancement of images, which employed an adaptive filter to control the contribution of the sharpening path so that contrast enhancement occurred in high detail areas and little or no image sharpening occurred in smooth areas. Guillon et al. [15] proposed an approach combining nonlinear low-pass and high-pass filters, which was also based on an adaptive filter mask and performed noise reduction as well as edge enhancement. Homomorphic filtering [2, 11, 35] was used for image enhancement. The image enhancement can be reduced to processing splitting-signals and such process requires a modification of only a few spectral components of the image, for each signal. Arslan and Grigoryan [3] proposed a fast implementation of the $\alpha$-rooting method by using one splitting- 
signal of the tensor representation with respect to the discrete Fourier transform. Greenspan et al. [14] described an image enhancement technique for enhancing the perceptual sharpness, which augmented the frequency content of the image using shape-invariant properties of edges across scale by using a nonlinearity that generates phase-coherent higher harmonics.

\section{The Description of the Algorithm}

\subsection{Improved Homomorphic Filtering}

Homomorphic filtering is a frequency domain filtering process that tries to separate the two components and enhance the reflectance while reduces the contributions of illumination [5]. In image processing, the possibility of optical implementation for the homomorphic filter makes it a powerful alternative to other enhancement techniques, such as histogram equalization. Figure 1 describes the homomorphic filtering [9].

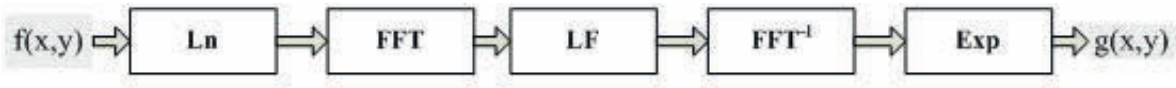

Figure 1. The flow chart of homomorphic filtering.

Usually, there are three second-order homomorphic high-pass filtering functions that are used as the linear filtering. The first one is the Gaussian filtering function, its expression is [33]

$$
H(u, v)=\left(r_{H}-r_{L}\right)\left(1-\exp \left[-c\left(\frac{\rho(u, v)}{\rho_{C}}\right)^{2}\right]\right)+r_{L} .
$$

The second one is the Butterworth filtering function defined as [1]

$$
H(u, v)=\left(r_{H}-r_{L}\right) /\left(1+\left(\frac{\rho(u, v)}{\rho_{C}}\right)^{2} / c\right)+r_{L} .
$$

The third one is the exponentiating filtering function that takes the form

$$
H(u, v)=\left(r_{H}-r_{L}\right)\left(1-\exp \left[\left(-c \frac{\rho(u, v)}{\rho_{C}}\right)^{2}\right]\right)+r_{L} .
$$

Among the above mentioned high pass filters, the Butterworth type is far superior to the other two frequency-domain filters. It can get the best result in dynamic range compression and contrast enhancement, which makes it suitable for the use with the homomorphic filtering approach [33]. It has global property and can keep 
overall appearance well for it makes use of all pixels in the image. However, it does not take local spatial characteristics into account and can not get satisfying results in image local contrast enhancement.

In order to enhance the local contrast of the image, we can modify the above Butterworth filtering function [23] and get

$$
H(\rho)=r_{1}-r_{2} \frac{1}{1+2.145\left(\frac{\rho}{\rho_{C}}\right)^{4}} .
$$

The parameters $r_{1}$ and $r_{2}$ were added for flexibility. By varying $r_{1}$ and $r_{2}$, one can obtain any value between $r_{1}$ and $r_{2}$. When $\frac{\rho}{\rho_{c}} \gg 1$, we can derive the relationship $r_{H} \approx r_{1}$; when $\frac{\rho}{\rho_{c}} \ll 1$, we can get $r_{L} \approx r_{1}-r_{2}$. Additionally, the sharpness of the transition between $r_{H}$ and $r_{L}$ can be controlled by varying the cutoff frequency $\rho_{C}$.

\subsection{Dyadic Wavelet Transform}

The wavelet analysis is developed in recent years, and it provides a multi-resolution representation of continuous and discrete-time signals and images. The advantage of this technique is that structures of different size appear at different scales and can be process independently [18]. Smooth symmetrical or anti symmetrical wavelet transform functions can be used, allowing alleviation of boundary effects via mirror extension of the signal. Due to these advantages, such representations have been extensively used in image enhancement. In this paper, a fast biorthogonal, redundant discrete wavelet transform based on a wavelet function $\psi(x)$ with compact support is utilized in our algorithm.

Let $\varsigma_{s}(x, y)=\left(1 / s^{2}\right) \varsigma(x / s, y / s)$ denote the dilation of a 2D function $\varsigma_{s}(x, y)$ at scale $s$. Any 2D function $f(x, y)$ can be approximated at a scale $s=2^{j}$ by an approximated operator $S_{2^{j}}$, defined by a convolution with a dilated scaling function: $S_{2^{j}} f(x, y)=f^{*} \varphi_{2^{j}}(x, y)$. The scaling function $\varphi(x, y)$ is a low pass function whose Fourier transform is an aggregation of wavelet functions dilated by scales than 1 . Any discrete image $I_{n, m}$ can be considered as the uniform sampling of the approximation of a function $f(x, y)$ at the finest measured scale 1 : $S_{1} f(m, n)=I_{n, m}$. The discrete wavelet transform is then a uniform sampling of the corresponding continuous wavelet transform, discretized over the scale parameter $s$ at dyadic scales $2^{j}$. For a particular class of spline wavelet functions, the 
wavelet transform of $f(x, y)$ has two components and the following form [22]:

$$
\begin{aligned}
\left\{\begin{array}{l}
W_{2^{j}}^{1}(x, y) \\
W_{2^{j}}^{2}(x, y)
\end{array}\right\} & =2^{j}\left\{\begin{array}{l}
f^{*} \psi_{2^{j}}^{1}(x, y) \\
f^{*} \psi_{2^{j}}^{2}(x, y)
\end{array}\right\} \\
& =2^{j}\left\{\begin{array}{c}
\frac{\partial}{\partial x}\left(f^{*} \theta_{2^{j}}^{1}(x, y)\right) \\
\frac{\partial}{\partial y}\left(f^{*} \theta_{2^{j}}^{2}(x, y)\right)
\end{array}\right\}=2^{j} \cdot \vec{\nabla}\left(f^{*} \theta_{2^{j}}\right)(x, y) .
\end{aligned}
$$

Here the analysis wavelets $\psi^{1}(x, y)$ and $\psi^{2}(x, y)$ are partial derivates of a symmetrical smoothing function $\theta(x, y)$, approximating the Gaussian and the dyadic scale $j$. Then the original image is decomposed into a multiresolution hierarchy of subband images, consisting of a coarse approximation image $S_{2^{j}} f(m, n)$ and a set of wavelet images $\left\{W_{2^{j}}^{1}(x, y), W_{2^{j}}^{2}(x, y)\right\}(j=1, \ldots, J)$. They provide the details that are available in $S_{1} f$ but disappeared in $S_{2^{j}} f$. All subband images have the same number of pixels as the original, thus the representation is highly redundant. At dyadic scale $j$, the discrete filters are $L H_{j}, H L_{j}, H H_{j}$ obtained by inserting $2^{j}-1$ zeros between each of the coefficients of the corresponding filters. Each level is composed by $L H_{j}, H L_{j}, H H_{j}$ high frequencies in the horizontal, vertical and diagonal direction as well as a low frequencies $L L_{j}$ in the highest level [30]. $L L_{j}$ reflects the illumination distribution and basic information in spatial domain. The wavelet coefficients under different resolution can be similarly high-pass processed to attenuate the low frequencies and amplify the high frequencies for the spatial characteristic of wavelet transform. And this spatial characteristic reflects the global information of the image to some extent.

\subsection{The New Image Enhancement Algorithm}

Unlike the basic homomorphic filtering methods that implement filtering on frequency domain, we present a new image enhancement algorithm that performs homomorphic filtering on spatial domain. Our method performs filtering on subband images of wavelet transform by using the improved Butterworth filtering. Meanwhile, different wavelet sub-band coefficients are enhanced differently. The flow chart in Figure 2 introduces the procedure of our method.

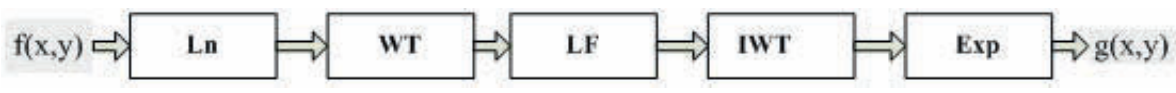

Figure 2. The flow chart of wavelet homomorphic filtering transform.

Here WT stands for wavelet transform, IWT is the inverse wavelet transform and LF represents the new high-pass filtering. 
The improved Butterworth filtering is a high-pass filter, and the low frequency is participated in the next iteration. As the number of decompositions increases, the high frequencies between two neighboring level decrease. So we can use larger filtering function values in contrasting higher level. In this paper, we set $r_{1}$ to be $j /(j+1)$ and $r_{2}$ to be $2 j / 5(j+1)$ (here $j$ is the decomposition level and $j=1, \ldots, J)$. We get $r_{1}$ and $r_{2}$ of the highest level to calculate $H_{L L_{j}}$. Here $r_{11}<r_{12}<\cdots<r_{1 J}$ and $r_{21}<r_{22}<\cdots<r_{2 J}$.

For $L H_{j}$ and $H L_{j}$, we set

$$
\frac{\rho}{\rho_{C}}=\sigma^{2} / \sum_{u=1}^{M} \sum_{v=1}^{N}\left[\left(u-u_{0}\right)^{2}+\left(v-v_{0}\right)^{2}\right] .
$$

Here both $M$ and $N$ are the size of the $j$ level sub-band, $\delta$ is the standard deviation and we get

$$
H(\rho)=r_{1}-r_{2} /\left(1+2.145\left(\sigma^{2} / \sum_{u=1}^{M} \sum_{v=1}^{N}\left[\left(u-u_{0}\right)^{2}+\left(v-v_{0}\right)^{2}\right]\right)^{4}\right) .
$$

For $H H_{j}$, we set $\frac{\rho}{\rho_{c}}=1 /\left(2^{j} \cdot k_{b}\right)$, here $k_{b}$ are the cutoff coefficients. In this paper, $k_{b}$ is set to be $1 / 8$.

Thus, the improved Butterworth filtering function is

$$
H(\rho)=r_{1}-r_{2} /\left(1+2.145\left(\frac{1}{2^{j} k_{b}}\right)^{4}\right) .
$$

For $L L_{N}$, the above equation can be written as $r_{1}-r_{2}$ and it can be processed by linear or nonlinear adjust to revise its illumination non-uniformity. We use the following equation to adjust it:

$$
H_{L L_{N}}=\left(r_{1}-r_{2}\right)(k(x-m)+m) .
$$

Here $m$ is the mean of the coefficient of $L L_{N}, x$ is the coefficient of $L L_{N}, k$ is the regulatory factor. When $k=1$, the high frequency is amplified but the illumination non-uniformity in the spatial domain remains very obviously, it can remain the original appearance of the image well. When $k=0$, the illumination non-uniformity in the spatial domain can be eliminated but original appearance of the image has been changed greatly for the low frequencies of $L L_{N}$ has been completely removed. These low frequencies are not entirely caused by the illumination non-uniformity. So $k$ should not be set as 0 or 1 . In this paper, we set $k=1-1 / m$ to adjust the local contrast and keep the original appearance of the image. 
In our work, we use this improved function as the linear filtering function. This new image enhancement algorithm performs in following steps: At first, an image is decomposed by dyadic wavelet transform. Afterwards, homomorphic filtering is performed on wavelet coefficients of the image. Here different sub-band images are processed differently through high-pass filter or linear adjust to attenuate low spatial frequencies and amplify high spatial frequencies. Finally, we utilize inverse dyadic wavelet transform to reconstruct the enhanced image.

\section{Experimental Results}

To demonstrate the performance of our algorithm, we carry out several experiments on a number of low contrast images and compare our method with representative image enhancement algorithms, such as the AHE method, the improved Butterworth filtering (IBF) method, and the directional wavelet transform (DWT) method. Here we use three level dyadic wavelet transform, and the cutoff frequency $\rho_{C}$ is set to be 80 in the improved Butterworth filtering method. In each of the Figures 3-10, the five images represent (a) the original image, (b) the result of the AHE method, (c) the result of the IBF method, (d) the result of the DWT method and (e) the result of our method.

\section{Discussion and Analysis}

From these experiments, we can see that as the original images have large homogeneous regions, the AHE method fails in its enhancing for it has been greatly magnify noise in smooth regions and the brightness has been changed greatly, which makes the enhanced image look rather unnatural. Meanwhile, the improved Butterworth filtering method outperforms the AHE method and there also exists change in brightness. The DWT method can obtain the best result among these representative methods.

Now, we focus on comparison of our method with the DWT method. From Figures 3-10, we can see that the image using our method is much clearer and presents more subtle tissue that can not be shown by the DWT method. In each figure, comparison of both methods illuminates that our method can get more exact and concrete tissues. Some tissue have not been correctly enhanced by the DWT method, while our method can keep them well. Table 1 gives the entropy comparison of each image shown in Figures 3-10. 


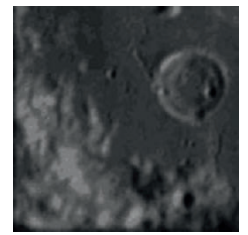

(a)

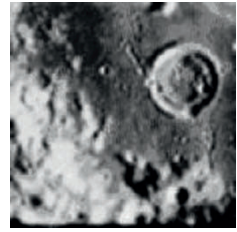

(b)

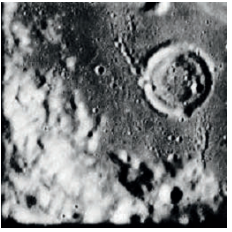

(c)

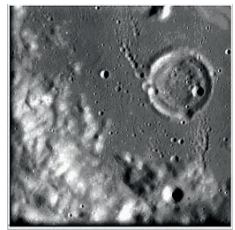

(d)

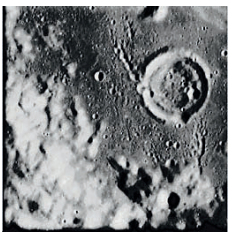

(e)

Figure 3

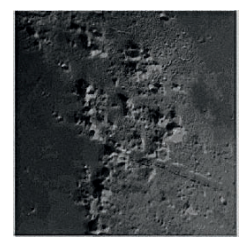

(a)

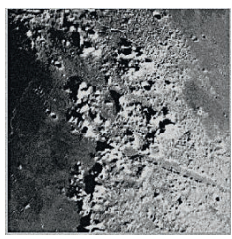

(b)

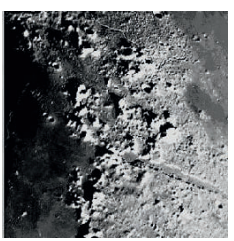

(c)

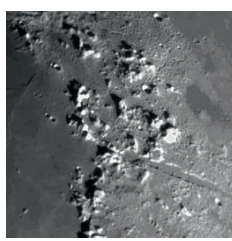

(d)

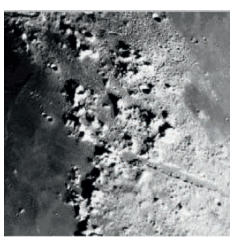

(e)

Figure 4

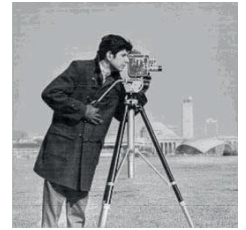

(a)

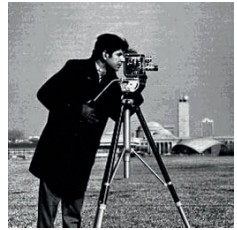

(b)

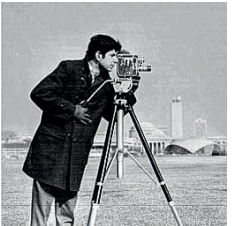

(c)

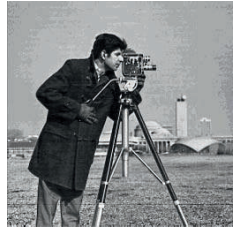

(d)

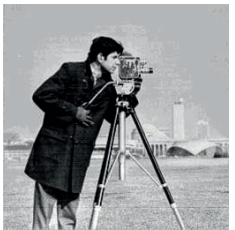

(e)

Figure 5

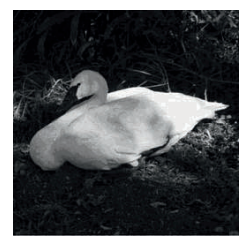

(a)

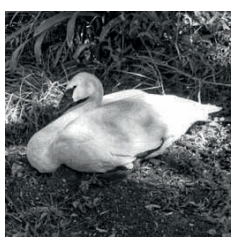

(b)

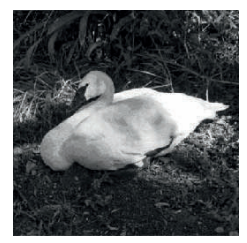

(c)

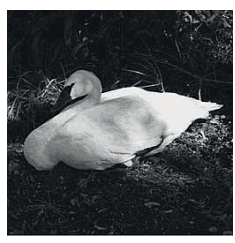

(d)

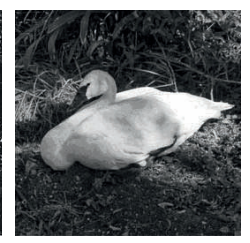

(e)

Figure 6 


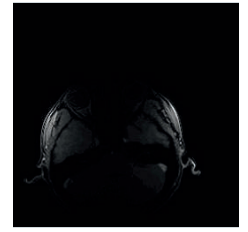

(a)

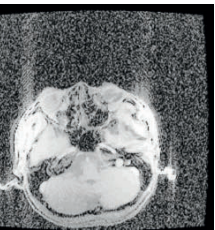

(b)

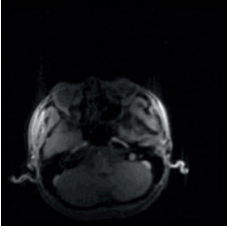

(c)

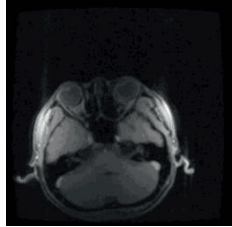

(d)

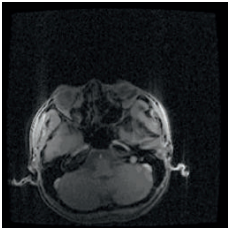

(e)

Figure 7

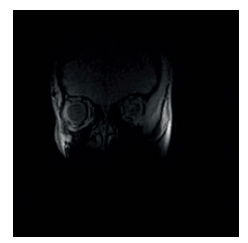

(a)

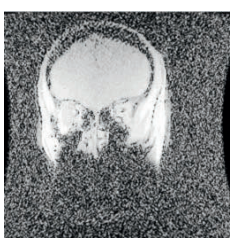

(b)

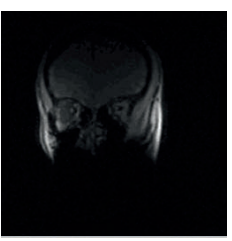

(c)

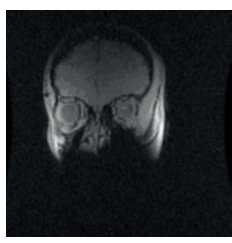

(d)

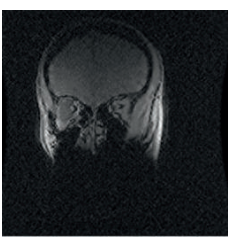

(e)

Figure 8

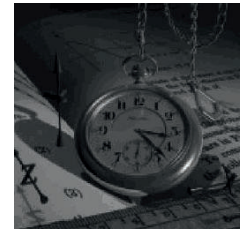

(a)

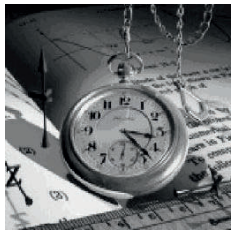

(b)

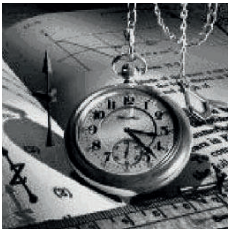

(c)

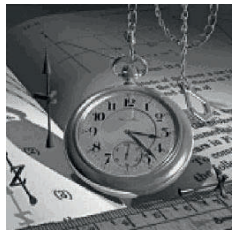

(d)

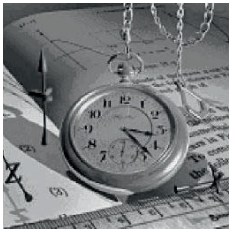

(e)

Figure 9

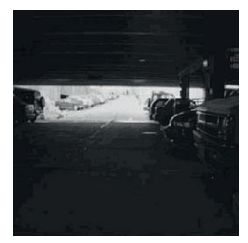

(a)

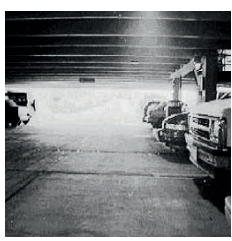

(b)

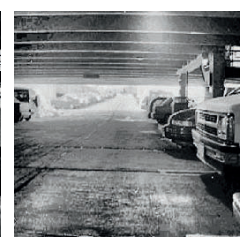

(c)

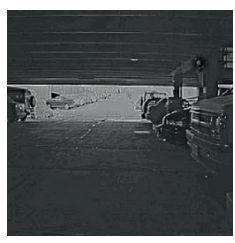

(d)

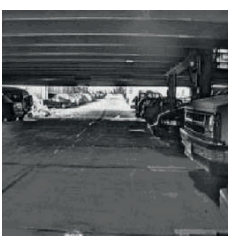

(e)

Figure 10 


\begin{tabular}{cccccc}
\hline & $\begin{array}{c}\text { Original } \\
\text { image }\end{array}$ & $\begin{array}{c}\text { AHE } \\
\text { method }\end{array}$ & $\begin{array}{c}\text { IBF } \\
\text { method }\end{array}$ & $\begin{array}{c}\text { DWT } \\
\text { method }\end{array}$ & $\begin{array}{c}\text { Proposed } \\
\text { method }\end{array}$ \\
\hline Figure 3 & 3.3048 & 1.8673 & 2.7307 & 3.0161 & 3.3157 \\
Figure 4 & 7.0383 & 3.4748 & 5.2127 & 5.5886 & 7.0796 \\
Figure 5 & 6.5493 & 2.5414 & 5.0112 & 5.2183 & 6.5502 \\
Figure 6 & 6.8272 & 2.6575 & 5.0307 & 5.4322 & 6.8404 \\
Figure 7 & 4.7884 & 1.8154 & 4.0113 & 4.3106 & 4.8109 \\
Figure 8 & 4.8868 & 1.9643 & 4.0857 & 4.2838 & 4.9083 \\
Figure 9 & 6.8109 & 2.6371 & 5.0120 & 5.2597 & 6.8310 \\
Figure 10 & 5.3748 & 2.1758 & 4.2032 & 5.0142 & 5.4164 \\
\hline
\end{tabular}

Table 1. Comparison of entropy.

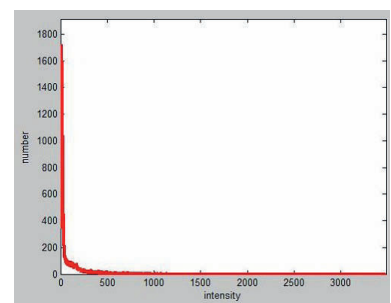

(a) Histogram of Figure 7 (a)

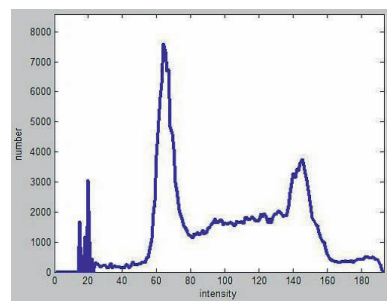

(b) Histogram of Figure 7 (d)

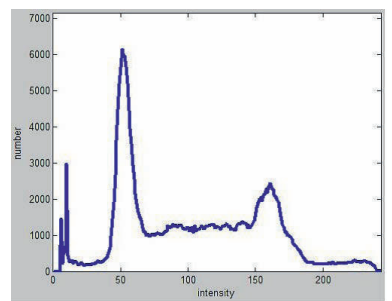

(c) Histogram of Figure 7 (e)

Figure 11. Corresponding 1D histogram of Figure 7 (a), (d) and (e).

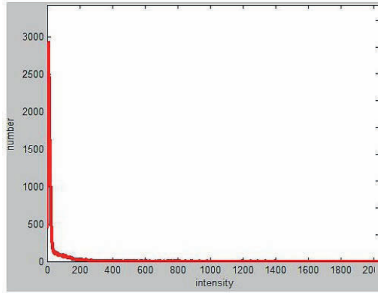

(a) Histogram of Figure 8 (a)

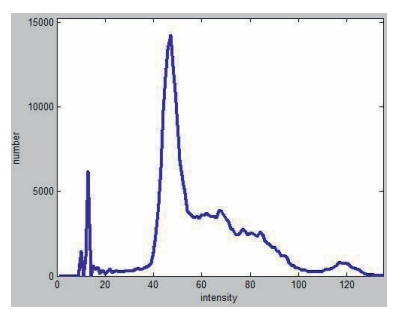

(b) Histogram of Figure 8 (d)

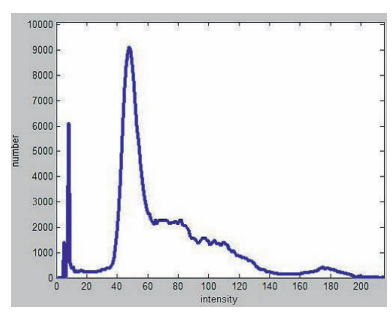

(c) Histogram of Figure 8 (e)

Figure 12. Corresponding 1D histogram of Figure 8 (a), (d) and (e). 
From Table 1 we can see that our method can keep the entropy well in contrast to the original image, while all other methods lose certain information to some extent.

On the basis of Figures 7 and 8, we compare the image 1D histogram of our method with the DWT method. Figure 11 is the corresponding histogram of Figure 7 (a), (d) and (e), while Figure 12 is the corresponding histogram of Figure $8(\mathrm{a}),(\mathrm{d})$ and (e).

From the corresponding histograms of the DWT method and our method, we can see that the dynamic range obtained by our method is much wider than the DWT method, which proves better ability of our method in contrast enhancement. This also explains that larger contrast can be obtained by our method.

\section{Conclusions}

In order to improve the contrast of different types of images for further analysis, we have given a spatial homomorphic filtering algorithm for image contrast enhancement. Images are firstly decomposed by wavelet transform and then different homomorphic filtering is implemented on the images. Experiments on different types of images, such as the remote sensing images, medical images, have shown that our method performs well in enhancing the local contrast of an image while maintaining its global appearance. Its performance has been proved to be much better than those representative methods and it may provide some new solutions for existing problems. Though this algorithm performs well in processing gray images, there will be some limitations in processing color images for it hasn't concerned much about the hue and saturation properties, which are the basic and important factors in color image enhancement. In the next period of research, we will concern more about the color images with this algorithm, for there are some techniques to be improved to some extent.

Acknowledgments. We are grateful to the anonymous referees for useful comments and suggestions.

\section{Bibliography}

[1] H. G. Ademanm, A frequency-domain Gaussian filter module for quantitative and reproducible a high-pass, low-pass filtering of images, American Laboratory 29 (1997), 27-33.

[2] H. G. Adelmann, Butterworth equations for homomorphic filtering of images, Computers in Biology and Medicine 28 (1998), 169-181. 
[3] F. T. Arslan and A. M. Grigoryan, Fast splitting a-rooting method of image enhancement: Tensor representation, IEEE Transactions on Image Processing 15 (11) (2006), 3375-3384.

[4] A. Beghcladi and A. L. Negrate, Contrast enhancement technique based on local detection of edges, Computer Vision, Graphics, and Image Processing 46 (1989), $162-174$.

[5] C. A. Castaño-Moraga, C.-F. Westin and J. Ruiz-Alzola, Homomorphic filtering of DT-MRI fields, The 6th International Conference on Medical Image Computing and Computer-Assisted Intervention (2003), 990-991.

[6] K. Castleman, Digital Image Processing, Prentice-Hall, Englewood Cliffs, 1996.

[7] Q. Chen et al., A solution to the deficiencies of image enhancement, Signal Processing 90 (2010), 44-56.

[8] S. D. Chen and A. R. Ramli, Minimum mean brightness error bihistogram equalization in contrast enhancement, IEEE Transactions on Consumer Electronics 49 (2003), 1310-1319.

[9] K. Dealc, M. Grgic and T. Kos, Sub-image homomorphic filtering technique for improving facial identification under difficult illumination conditions, International Conference on Systems, Signals and Image Processing 1 (2006), 21-23.

[10] R. Fattal, D. Lischinski and M. Werman, Gradient domain high dynamic range compression, in: Proceedings of the ACM SIGGRAPH (2002), 249-256.

[11] R. Fries and J. Modestino, Image enhancement by stochastic homomorphic filtering, IEEE Transactions on Acoustics, Speech, and Signal Processing 27 (1979), 625-637.

[12] B. Funt, F. Ciurea and J. McCann, Retinex in Matlab, Journal of the Electronic Imaging 13 (2004), 48-57.

[13] G. Gilboa, N. Sochen and Y. Y. Zeevi, Image enhancement and denoising by complex diffusion processes, IEEE Transactions on Pattern Analysis and Machine Intelligence 26 (2004), 1020-1036.

[14] H. Greenspan, C. H. Anderson and S. Akber, Image enhancement by nonlinear extrapolation in frequency space, IEEE Transactions on Image Processing 9 (2000), 1035-1048.

[15] S. Guillon, P. Baylou, M. Najim and N. Keskes, Adaptive nonlinear filters for 2D and 3D image enhancement, Signal Processing 67 (1998), 237-254.

[16] T. L. Ji, M. K. Sundareshan and H. Roehrig, Adaptive image contrast enhancement based on human visual properties, IEEE Transactions on Medical Imaging 13 (1994), 573-586.

[17] D. J. Jobson, Z. Rahman and G. A. Woodell, A multiscale Retinex for bridging the gap between color images and the human observation of scenes, IEEE Transactions on Image Processing 6 (1997), 965-976. 
[18] S. Y. Kim, Image contrast enhancement based on the piecewise linear approximation of CDF, IEEE Transactions on Consumer Electronics 45 (1999), 828-834.

[19] R. Kimmel, M. Elad, D. Shaked, R. Keshet and I. Sobel, A variational framework for Retinex, International Journal of Computer Vision 52 (2003), 7-23.

[20] E. Land, The Retinex, American Scientist 52 (1964), 247-264.

[21] E. Land and J. McCann, Lightness and Retinex theory, Journal of the Optical Society of America 61 (1971), 1-11.

[22] J. Liu and P. Moulin, Information-theoretic analysis of inter-scale and intra-scale dependencies between image wavelet coefficients, IEEE Transaction on Image Processing 10 (2001), 1647-1658.

[23] I. V. Liviu, R. M. Harley and R. W. Arthur, Practical considerations on color image enhancement using homomophic filtering, Journal of Electronic Imaging 6 (1997), 108-113.

[24] A. Majumder and S. Irani, Perception-based contrast enhancement of images, $A C M$ Transactions on Applied Perception 4 (2007), 1-22.

[25] S. Marsi, G. Impoco, A. Ukovich, G. Ramponi and S. Carrato, Using a recursive rational filter to enhancement color images, IEEE Transactions on Instrumentation and Measurement 57 (2008), 1230-1236.

[26] L. Meylan and S. Susstrunk, High dynamic range image rendering with a Retinexbased adaptive filter, IEEE Transactions on Image Processing 15 (2006), 2820-2830.

[27] K. A. Panetta, E. J. Wharton and S. S. Agaian, Human visual system-based image enhancement and logarithmic contrast measure, IEEE Transactions on Systems, Man, and Cybernetics, Part B: Cybernetics 38 (2008), 174-188.

[28] S. M. Pizer et al., Adaptive histogram equalization and its variation, Computer Vision, Graphics, and Image Processing 39 (1987), 355-368.

[29] A. Polesel, G. Ramponi and V. J. Mathews, Image enhancement via adaptive unsharp masking, IEEE Transactions on Image Processing 9 (2000), 505-510.

[30] Z. H. Qi et al., X-ray image enhancement based on the dyadic wavelet transform, Journal of X-Ray Science and Technology 14 (2006), 83-93.

[31] D. H. Rao and P. P. Panduranga, A survey on image enhancement techniques: Classical spatial filter, neural network, cellular neural network, and fuzzy filter, in: IEEE International Conference on Industrial Technology (2006), 2821-2826.

[32] G. Sapiro and V. Caselles, Contrast enhancement via image evolution flows, Graphical Models and Image Processing 59 (1997), 407-416.

[33] M. J. Seow and V. K. Asart, Ratio rule and homomorphic filter for enhancement of digital color image, Neurocomputing 69 (2006), 954-958. 
[34] J. Tumblin and G. Turk, LCIS: A boundary hierarchy for detail-preserving contrast reduction, in: Proceedings of the SIGGRAPH Annual Conference on Computer Graphics (Los Angeles 1999), 83-90.

[35] L. I. Voicu, H. R. Myler and A. R. Weeks, Practical considerations on color image enhancement using homomorphic filtering, Journal of Electronic Imaging 6 (1997), $108-113$.

[36] D. Yi and S. Lee, Fourth-order partial differential equations for image enhancement, Applied Mathematics and Computation 175 (2006), 430-440.

Received August 6, 2012.

\section{Author information}

Chao Li, College of Electronics and Information, Tongji University, Shanghai, 201804, P. R. China.

E-mail: lic321@163.com

Guangyao Li, College of Electronics and Information, Tongji University, Shanghai, 201804, P. R. China.

E-mail: lgy@tongji.edu.cn

Yunlan Tan, College of Electronics and Information, Tongji University, Shanghai, 201804; and College of Electronics and Information, Jinggangshan University, Jiangxi, 343009, P. R. China.

E-mail: tanyunlan@163.com

Xianglong Xu, College of Electronics and Information, Tongji University, Shanghai, 201804, P. R. China.

E-mail: xux16125@163.com

Huixian Duan, College of Electronics and Information, Tongji University, Shanghai, 201804, P. R. China.

E-mail: hxduan@tongji.edu.cn 